\title{
Modified technique for common carotid artery transposition in standing horses
}

\section{Tapio, Heidi}

\author{
2017-01
}

Tapio , H , Argüelles Capilla , D , Gracia-Calvo , L A \& Raekallio , M 2017 , ' Modified technique for common carotid artery transposition in standing horses ' , Veterinary Surgery , vol. 46 , no. 1 , pp. 52-58 . https://doi.org/10.1111/vsu.12585

http://hdl.handle.net/10138/311056

https://doi.org/10.1111/vsu.12585

cc_by_nc

acceptedVersion

Downloaded from Helda, University of Helsinki institutional repository.

This is an electronic reprint of the original article.

This reprint may differ from the original in pagination and typographic detail.

Please cite the original version. 
1 Title page

2

3 The running head: Modified technique for common carotid artery transposition in

$4 \quad$ standing horses

5

6 The article title: Modified technique for common carotid artery transposition in standing

7 horses

8

9 Authors: Heidi Tapio DVM, David Argüelles DVM PhD Dipl ECVS, Luis A. Gracia-

10 Calvo DVM MS PhD Dipl ECVS, Marja Raekallio DVM PhD

11

12 University of Helsinki, Faculty of Veterinary Medicine, Department of Equine and Small

13 Animal Medicine, Helsinki, Finland

14

15 Corresponding author: Heidi Tapio

16 Address: Veterinary Teaching Hospital, PL 57, 00014 University of Helsinki, Finland

17 E-mail: $\underline{\text { Heidi.tapio@helsinki.fi }}$

18 
19 Abstract

21 Objectives: To describe a modified technique for permanent translocation of the common

22 carotid artery to a subcutaneous position in standing horses.

24 Study design: Experimental study

26 Animals: Eight clinically healthy, adult Standardbred and Warmblood horses.

28 Methods: The surgery was performed with the horses standing under sedation and with

29 local anesthesia. A combination of previously described techniques was used modifying

30 the approach and closure of the incision. The right common carotid artery was

31 approached through a linear skin incision dorsal and parallel to the jugular vein and

32 through the brachiocephalicus and omohyoideus muscles. The artery was dissected free

33 of its sheath and elevated to the skin incision with Penrose drains. The brachiocephalicus

34 muscle was sutured in 2 layers underneath the artery leaving it in a subcutaneous

35 position. The horses were allowed to heal for 3 weeks prior to catheterization of the

36 artery.

38 Results: The transpositioned common carotid artery was successfully used for repeated 39 catheterization in 6 out of 8 horses for a period of 10 weeks. None of the horses had 40 intraoperative complications. Two horses developed mild peri-incisional edema that 41 resolved spontaneously. Right-sided laryngeal hemiplegia was observed endoscopically 42 in 2 horses postoperatively. Two horses developed complications (surgical site infection 
43 and excessive periarterial fibrosis, respectively) that compromised the patency of the

44 common carotid artery and precluded the catheterization.

45

46 Conclusions: The permanent translocation of the common carotid artery in a standing

47 horse was successful in 6 out of 8 horses. An upper airway endoscopy postoperatively

48 may be warranted as laryngeal hemiplegia may ensue.

49

50

51

52

53

54

55

56

57

58

59

60

61

62

63 
66 Repeated sampling of arterial blood and direct arterial blood pressure monitoring are 67 required in many physiologic and pharmacologic experiments in animal models. The 68 common carotid artery is frequently translocated to a subcutaneous position to facilitate 69 repeated sampling or catheterization of the artery in large animals, such as sheep, cattle, 70 and horses. ${ }^{1-6}$ Different techniques have been described for subcutaneous translocation of

71 the common carotid artery in anesthetized small ruminants. ${ }^{1,3,6}$ In horses, the available

72 literature on the procedure is scarce and is described for anesthetized horses. ${ }^{2,5,7} \mathrm{~A}$

73 technique to translocate the common carotid artery in the horse was first described by

74 Tavernor et al. $^{2}$ The common carotid artery was approached through a linear skin

75 incision between the jugular vein and the brachiocephalicus muscle. The omohyoideus

76 muscle was split to access the carotid sheath. The common carotid artery was elevated to

77 the skin incision and supported subcutaneously by reapposing the omohyoideus muscle

78 and with polyethylene tubing. The technique has subsequently been modified, including

79 the creation of a skin flap over the jugular groove ${ }^{5,7}$ and approach through the

80 brachiocephalicus muscle instead of accessing the carotid sheath ventral to it. ${ }^{7}$ The

81 previously described technique involving the creation of a skin flap reported formation of

82 seroma as complication in cases where suction drainage system was not utilized

83 postoperatively. ${ }^{5}$ In sheep and goats the reported complications of the permanent

84 translocation of the common carotid artery include subcutaneous hematoma and abscess

85 formation, occlusion of the elevated artery segment and necrosis of the skin flap. ${ }^{6}$ 
88 A surgical approach, similar to ours, has been previously described for temporary

89 catheterization of the common carotid artery in a standing horse for occlusion of the

90 internal carotid artery for the treatment of guttural pouch mycosis. ${ }^{8}$ After coil

91 embolization, the common carotid artery was released back to its deep location and

92 muscle layers were reapposed. The technique was successful in standing horses and no

93 perioperative complications occurred. However, this technique is only applicable for

94 single catheterization of the artery.

96 The purpose of this article is to describe a modified technique for the permanent

97 translocation of the common carotid artery to a subcutaneous position in the standing

98 horse. The surgical approach combined the previously described linear skin incision ${ }^{2}$ and

99 approach through the brachiocephalicus muscle ${ }^{7}$. Use of a polyethylene tubing or

100 postoperative suction system were omitted. In addition, method to reappose of the

101 muscles layers underneath the translocated common carotid artery was modified.

102

103 Performing the surgery standing would avoid the costs and potential risks related to the

104 general anesthesia of a horse. To the best of our knowledge, the permanent translocation

105 of the common carotid artery has not previously been described in a standing horse. 
Materials and methods

111

112 The study was approved by the National Animal Experiment Board of Finland and

113 conducted according to the Finnish Act on Animal Experimentation. This is consistent

114 with the U.S. National Institutes of Health "Guide for the Care and Use of Laboratory 115 Animals".

116

117 Eight healthy Standardbred and Warmblood horses (3 geldings and 5 mares) of ages 6-

11815 years were used in the study. The horses showed no signs of systemic illness based on

119 clinical examination and hematological analysis of blood samples.

120

121 The surgery was carried out with the horses standing and restrained in stocks. The head

122 was supported to remain at the level of the withers by cross-tying the horse. All the

123 procedures were performed on the right common carotid artery. A 12G

124 polytetrafluoroethylene intravenous catheter was placed aseptically into the left jugular

125 vein. All the horses were preoperatively medicated with penicillin G procaine 20,000

$126 \mathrm{IU} / \mathrm{kg}$ intramuscularly [IM] and flunixin meglumine $1.1 \mathrm{mg} / \mathrm{kg}$ intravenously [IV].

127 Sedation was accomplished by intravenous injection of detomidine hydrochloride (5

$128 \mu \mathrm{g} / \mathrm{kg}$ ) and butorphanol tartrate $(5 \mu \mathrm{g} / \mathrm{kg})$, and maintained either by continuous rate

129 infusion of detomidine hydrochloride $(30 \mu \mathrm{g} / \mathrm{kg} / \mathrm{hr})$ or repeated boluses of detomidine

130 hydrochloride $(2-3 \mu \mathrm{g} / \mathrm{kg}$ ) IV to effect. Boluses of detomidine hydrochloride were given

131 if horses started to move the head during the surgery. 
133 On the right side of the neck, the hair was clipped on the proximal and middle thirds,

134 continuing from the ventral midline of the neck approximately 20 centimeters dorsally.

135 The clipped area was aseptically prepared for surgery; the skin was scrubbed with

136 chlorhexidine gluconate skin cleanser and then wiped with alcohol solution. The surgical

137 area was draped sterilely.

139 Local anesthesia $(12-15 \mathrm{~mL}$ of lidocaine $2 \mathrm{mg} / \mathrm{mL})$ was infiltrated at the surgical site

140 subcutaneously on the dorsal aspect of the jugular groove prior to the start of the

141 operation. Additional local anesthesia ( $5 \mathrm{~mL}$ of lidocaine $2 \mathrm{mg} / \mathrm{mL}$ ) was infiltrated in the

142 brachiocephalicus and omohyoideus muscles if needed during the operation.

144 The skin was placed under tension dorsally using a sterile hand dorsal to the jugular

145 groove. A $10-12 \mathrm{~cm}$ linear skin incision was made with a number 22 blade at the

146 junction of the proximal and middle third of the neck, starting approximately $15 \mathrm{~cm}$

147 distal to the ramus of the mandible. The incision was made approximately $2 \mathrm{~cm}$ dorsally

148 and parallel to the jugular groove, also incising the cutaneous coli muscle (Fig 1). The

149 edges of the incision were undermined approximately $10 \mathrm{~mm}$ superficial to fascia of the

150 brachiocephalicus muscle using Metzenbaum scissors to create space for later closure of

151 the muscle layers. The fascia of the brachiocephalicus muscle was sharply incised and

152 the brachiocephalicus muscle was bluntly split using Metzenbaum scissors. The

153 underlying omohyoideus muscle was identified and bluntly split, digitally or using

154 Metzenbaum scissors, until the carotid sheath was visible. Weitlaner retractors were used

155 to facilitate the visibility and dissection. 
157 The carotid sheath was bluntly opened with Halsted mosquito forceps and the common 158 carotid artery and vagosympathetic trunk were identified (Fig 2). The opening of the

159 carotid sheath was digitally extended proximally and distally until it was the same length

160 as the incision. The carotid artery was then, digitally and using Halsted mosquito forceps,

161 separated from the carotid sheath and the vagosympathetic trunk. If present, small

162 branches of the carotid artery were identified, double ligated using poliglecaprone 25

163 USP 2-0, and severed. Approximately $7 \mathrm{~cm}$ of the artery was dissected free from the

164 sheath, so that it could be relocated subcutaneously without excessive tension or

165 restriction by the carotid sheath. Two Penrose drains, one proximally and one distally,

166 were then placed around the carotid artery and secured with Halsted mosquito forceps.

167 The artery was elevated to the skin incision by pulling the drains. The incision was

168 carefully checked for possible bleeding vessels and the surgical area was flushed with

169 sterile saline.

170

171 The fascia of the omohyoideus muscle and the overlying, deep part of the

172 brachiocephalicus muscle were sutured with a simple interrupted pattern using

173 polydioxanone USP 0 or polyglactin 910 USP 0 . The superficial part of the

174 brachiocephalicus muscle and the superficial fascia of the brachiocephalicus muscle were

175 then closed with an interrupted horizontal mattress pattern using polydioxanone USP 0 or

176 polyglactin 910 USP 0, leaving the common carotid artery superficial to it (Fig. 3). The

177 mattress pattern sutures were placed a minimum of $5 \mathrm{~mm}$ away from the incision of the

178 fascia of the brachiocephalicus muscle. The Penrose drains were then released and the

179 surgical area was inspected for any bleeding. The surgical site was flushed with saline 180 prior to closure of the skin. 
182 The subcutis layer was separately closed in 1 horse using a simple continuous pattern

183 with polyglactin 910 USP 2-0. In all the other horses, the subcutis layer was not closed.

184 The skin was closed with either a simple interrupted or Ford interlocking pattern using 185 polyamide 6 , USP 2-0. A stent bandage of sterile gauze was sutured over the incision in 5

186 horses using polyamide 6, USP 2-0. The suture pattern consisted of 3 interrupted sutures

187 using a single bite in the skin on each side of the bandage. The stent bandage was left in 188 place for $24-48$ hours.

190 Postoperatively, penicillin G procaine 20,000 IU/kg IM twice daily and flunixin

191 meglumine $1.1 \mathrm{mg} / \mathrm{kg}$ orally once daily were continued for 3-5 days. Monitoring of the

192 horses included recording the general attitude, appetite, amount of defecation and

193 performing a clinical examination 3 times a day. The surgical area was monitored daily

194 for swelling, a visible pulse in the translocated common carotid artery segment, and

195 incisional discharge until removal of the skin sutures. The horses were fed from a hay net

196 during the first postoperative week. The skin sutures were removed 12-14 days

197 postoperatively. After the removal of the skin sutures, the pulse of the elevated artery

198 segment was also palpated. If any incisional discharge was noticed or edema remained

199 after removal of the skin sutures, an ultrasound examination was performed.

201 Observed complications were recorded. Incisional dehiscence, infection, hematoma or 202 seroma at the surgical site were considered as minor complications. Inability to use the 203 translocated common carotid artery segment for catheterization was considered as major 204 complication. 
206 The elevated arteries were considered to be ready for use after the operation when all the 207 edema had resolved, a pulse of the elevated artery segment was clearly palpable 208 subcutaneously at the surgical site, and the skin incision had completely healed.

210 Six out of the 8 horses had an upper airway endoscopy at rest performed at 3 months

211 postoperatively in order to obtain a bronchoalveolar sample related to another study. 
214 Results

215

216 All the horses were operated without complications during the surgery. The results are

217 summarized in Table 1. In 2 horses (\#6 and \#8), a branch of the common carotid artery

218 needed to be ligated and severed. In 2 horses (\#7 and \#8), hemorrhage occurred from the

219 brachiocephalicus muscle, which was controlled with ligation of the vessels.

220

221 Six out of the 8 horses were used for experimental purposes after translocation of the

222 common carotid artery. The right common carotid artery was repeatedly catheterized

223 (BD Arterial Cannula 20G/1.10 mm x $45 \mathrm{~mm}$ ) throughout a period of 10 weeks. The

224 elevated artery was ready to be used in 3 weeks in these 6 horses. Catheterization was

225 performed weekly according to the study design and a catheter was used for repeated

226 blood sampling, direct blood pressure monitoring, and measurement of cardiac output by

227 the lithium dilution method. A catheter was in place for a maximum of 3 hours and was

228 secured in place with sutures. No complications occurred during the use of the artery.

229

230 In 2 out of 8 horses (\#1 and \#7), mild, non-painful edema developed around the incision.

231 The edema spontaneously resolved within 6 days. Right-sided laryngeal hemiplegia was

232 observed in 2 out of the 6 horses (\#1 and \#5) in which an upper airway endoscopy was

233 performed 3 months after the surgery. The hemiplegia was subjectively graded as $3 \mathrm{C}$ in

234 horse \#1 and as 3B in horse \#5. ${ }^{9}$ The horses showed no clinical signs of airway disease at 235 rest. 
237 In 2 horses (\#3 and \#4), complications occurred that compromised patency of the 238 common carotid artery and precluded the use the affected artery in the subsequent

239 experiments. A surgical site infection occurred in 1 horse and excessive periarterial

240 fibrosis at the surgical site in another.

242 Horse \#3 developed mild edema around the incision during 24 hours after the operation.

243 The edema increased to a moderate amount at 3 days postoperatively and the incision

244 started to discharge serosanguineous fluid. The discharge became purulent 7 days after

245 the operation and an incisional infection was confirmed with bacterial culture of

246 Clostridium sp. and Enterococcus faecalis. On ultrasound examination, the common

247 carotid artery was patent proximal and distal to the incision, but not in the surgical area.

248 The use of color Doppler ultrasonography confirmed the absence of blood flow. There

249 was also abnormally thick heterogeneous tissue around the elevated carotid segment. The

250 distal skin sutures were removed and the incision was flushed daily with saline. The

251 horse received penicillin $\mathrm{G}$ procaine $(20,000 \mathrm{IU} / \mathrm{kg}$ IM twice daily) and flunixin

252 meglumine $(1.1 \mathrm{mg} / \mathrm{kg}$ orally once daily) for 1 week. The rest of the sutures were

253 removed 12 days postoperatively and local wound cleaning with saline continued. The

254 horse remained afebrile and without systemic signs of illness throughout the treatment

255 period, and the discharge from the incision resolved over 1 month. The follow-up

256 ultrasonographic examination 1 month post-operatively revealed that the common carotid

257 artery was no longer patent. The horse was excluded from the subsequent studies.

259 Horse \#4 developed moderate, non-painful, subcutaneous edema around the incision 260 postoperatively that resolved spontaneously in 6 days. The incision was clean and there 
261 was no discharge. Thirteen days postoperatively, moderate, non-painful, dense swelling 262 developed around the incision. The sutures were removed, and ultrasonographic 263 examination revealed a moderate amount of heterogeneous tissue surrounding the 264 common carotid artery beneath the incision. A marked reduction in the patency of the 265 artery at the site of the incision was observed with color Doppler ultrasonography. The 266 horse was excluded from the subsequent studies. 
Discussion

270 Transposition of the common carotid artery was carried out in all 8 standing horses

271 without intraoperative complications. Six of the 8 horses were successfully used in the

272 subsequent studies, in which the elevated artery segment was repeatedly catheterized.

274 An adequate level of sedation and analgesia was achieved in all the horses and the

275 procedure was well tolerated. Performing the transposition of the common carotid artery

276 with the horse standing avoids risks related to general anesthesia and the recovery of the

277 horse, while also reducing the time and costs of the procedure. The common carotid

278 artery was readily accessed through the muscle layers in a standing horse.

280 The technique described in this study is a modification of previously described

281 techniques to translocate the common carotid artery. ${ }^{1-3,5-6,10}$ In the present study, the

282 common carotid artery was approached through a linear skin incision, as described

283 earlier. ${ }^{2}$ Orsini and Roby presented an approach involving the creation of a skin flap over

284 the jugular groove. The skin flap was performed in order to facilitate tissue identification

285 and percutaneous puncture of the carotid artery. However, seroma formation was

286 described in 8/14 horses in which a closed suction system was not applied

287 postoperatively. ${ }^{5}$ We observed no seroma formation postoperatively, although suction

288 systems were not used. This could be related to the reduced dead space formation with

289 the technique we used compared to the creation of a flap. In addition, closure of the

290 muscles in 2 layers underneath the elevated carotid artery further reduces the dead space

291 and may assist in the prevention of a seroma. 
293 The carotid sheath has either been approached through the brachiocephalicus muscle, ${ }^{8,9}$

294 ventral to it, ${ }^{2,5}$ or through the sternocephalic muscle ventral to the jugular groove. ${ }^{10} \mathrm{We}$

295 chose to approach the common carotid artery directly through the brachiocephalicus and

296 omohyoideus muscles. Approach through the brachiocephalicus muscle involved

297 splitting of the muscle by blunt dissection, but provided a strong muscle fascia to be

298 sutured underneath the common carotid artery. Furthermore, the jugular vein was not at

299 risk of inadvertent puncturing during suturing, as it was located well away from the

300 incision. Care was taken when dissecting muscle to avoid excessive trauma to the muscle

301 and to close the dead space as much as possible in order to avoid seroma formation.

303 In the method described by Tavernor, the carotid artery segment was supported

304 subcutaneously with a polyethylene tube. ${ }^{2}$ In later modifications of the technique in

305 horses, including ours, it has been observed that no support is needed to keep the artery

306 elevated. ${ }^{5,7}$ The use of foreign material to support the arterial segment could increase the

307 risk of an excessive foreign body reaction, postoperative infection, and excessive fibrosis

308 through mechanical irritation of the arterial wall.

310 Periarterial fibrosis developed in 1 horse at the surgical site and resulted in reduced

311 patency of the elevated arterial segment. Occlusion of the artery by space-occupying

312 lesions, like periarterial hematoma or abscess, has been reported as complication in sheep

313 after permanent translocation of the common carotid artery. ${ }^{6}$ Fibrosis surrounding the

314 elevated artery in this horse may have led to stenosis by physical constriction and by

315 reducing the elasticity of the surrounding tissues, thus reducing the pulsatility of the 
316 artery. In addition, periarterial fibrosis complicates the catheterization of the artery even

317 if the patency remained normal. This complication occurred in the only horse in which

318 the subcutaneous tissues were closed with a separate layer, which could potentially

319 contribute to fibrosis formation because of the presence of additional foreign material at

320 the surgical site. Moreover, the artery should be dissected completely free of the

321 surrounding carotid sheath prior to elevation to the incision. Failure to do so could

322 potentially leave the carotid sheath constricting the elevated artery or result in excessive

323 stretching of the artery when its being elevated to the incision. These factors may

324 contribute to formation of fibrosis and consequently stenosis of the artery. In addition,

325 while suturing the muscles, it is important to ensure that enough space is left for the

326 artery segment to pass through muscles layers without constriction. The

327 brachiocephalicus muscle and its superficial fascia were closed with a horizontal mattress

328 pattern. A simple interrupted or continuous pattern may suffice to relieve the tension of

329 the incision. However, using a mattress pattern, no suture material is in direct contact

330 with the elevated artery, reducing the risk of inducing inflammation of the arterial wall

331 by mechanical irritation of the suture material.

333 Damage to the vagosympathetic trunk that runs within the carotid sheath, and the right

334 recurrent laryngeal nerve parallel to the trachea, should be avoided when elevating the

335 carotid artery free of its sheath. Two out of 6 horses showed signs of right-sided

336 laryngeal hemiplegia on endoscopic examination of the larynx postoperatively.

337 Postoperative airway endoscopy was not performed for the 2 horses that were excluded

338 from the subsequent studies. Laryngeal hemiplegia may be a consequence of damage to

339 the recurrent laryngeal nerve or to the vagus nerve within the vagosympathetic trunk. ${ }^{11}$ 
340 We did not observe other clinical signs related to the damage of the vagosympathetic

341 trunk, such as Horner's syndrome or dysfunction of the pharynx. ${ }^{11}$ Therefore, we believe

342 that damage to the recurrent laryngeal nerve was more likely than damage to the vagus

343 nerve in those cases. Endoscopy of the upper respiratory tract of the horses prior to

344 surgery was not performed. However, given the rarity of the condition on the right side, ${ }^{12}$

345 it is likely that surgical manipulation together with inflammation contributed to this.

346 Laryngeal hemiplegia has been reported as a potential complication after surgery in the

347 neck region. ${ }^{12}$ Loss of nerve function could occur through failure of impulse conduction

348 (type I injury) or through direct damage to the axonal structures (type II injury). ${ }^{13,14}$ Type

349 I injury, neurapraxia, results, for example, from excessive compression of the nerves,

350 which could be caused by postsurgical edema or damage to the myelin sheath, that may

351 be caused by the manipulation during the surgery. This type of nerve damage is usually

352 reversible, although it may persist months, in comparison to type II injury, in which

353 prognosis for recovery is poor and therefore nerve function may be permanently lost. ${ }^{14}$

354 Either type of injury of the right recurrent laryngeal nerve in this case is possible. A

355 damage due to surgical manipulation or inflammation seems plausible as an excessive 356 compression due to postoperative edema would also be likely to affect the

357 vagosympathetic trunk, as it is very closely located. Careful tissue manipulation is

358 needed to avoid surgical trauma to the nerves and to reduce postoperative inflammation.

359 In addition, the use of non-steroidal anti-inflammatory medication peri- and

360 postoperatively could aid in reducing later surgical site inflammation. However, as the

361 number of horses in our study was low and the postoperative status of the larynx in 2

362 horses remained unknown, the true risk of laryngeal hemiplegia following this procedure

363 should be further investigated with a larger number of horses. 
365 In the present study, surgical site infection occurred in 1 horse, which led to occlusion of

366 the elevated artery segment and precluded the use of the horse in cardiovascular studies.

367 The reason for the surgical site infection was unknown. Strict asepsis was followed

368 during the surgery and there were no intraoperative complications, such as excessive

369 hemorrhage. However, performing the surgery standing in an examination room in

370 comparison to operating on an anesthetized horse in a surgery room could potentially

371 expose the surgical site to contamination. A stent bandage was not used postoperatively

372 in this horse, which may have exposed the incision to contamination or contributed to the

373 formation of edema that preceded the infection. Covering the incision for example with a

374 stent bandage or a sterile wrap protects the incision while the initial seal of the skin

375 occurs. In addition, it may help to prevent the formation of a hematoma and/or seroma.

377 An ultrasound-guided technique to catheterize the common carotid artery in its deep

378 location has also been described. ${ }^{15,16}$ This technique avoids the surgical transposition of

379 the common carotid artery, but the catheterization needs to be assisted with

380 ultrasonography. We believe that a subcutaneously located common carotid artery is

381 more readily catheterized repeatedly, although this requires surgery prior to the start of

382 the experiment. Furthermore, if several catheters are simultaneously used in jugular veins

383 in addition to the arterial catheter, the subcutaneous location of the carotid artery in the

384 proximal part of the neck may facilitate catheterization without compromising the jugular 385 veins. 
387 After a permanent transposition of the common carotid artery the horses may have a

388 higher risk for fatal hemorrhage if they sustain a laceration to the right side of the neck at

389 the site of the surgery. However, we have observed no complications related to the

390 subcutaneous location of the artery and the horses were managed and turned out

391 normally. The pulse of the translocated common carotid artery has remained visible and

392 palpable subcutaneously 24 months postoperatively in the two horses that are is still in

393 the hospital herd. In order to decrease the risk for fatal hemorrhage in the event of a neck

394 laceration, a reversal of the common carotid artery back to its deep location could be

395 considered. However, the benefits and risks of such operation remain to be investigated.

397 In our study, the translocated artery segment was successfully catheterized for a period of

39810 weeks in 6 horses. During this time period, we encountered no difficulties in

399 catheterization of the artery and observed no changes in cardiovascular parameters of the

400 horses. This is comparable with a previous report in which a common carotid artery

401 segment was translocated subcutaneously under general anesthesia in horses and

402 successfully used for 12 weeks. $^{7}$ However, the long-term results of the procedure in 403 horses should be determined with a study including a longer follow-up period.

405 The permanent translocation of the common carotid artery can be performed in a 406 standing horse and it was well tolerated by the horses. It is an alternative to performing

407 the procedure under general anesthesia, as the potential risks of anesthesia and recovery 408 of the horse can be avoided while also reducing the costs of the surgery. An upper airway 409 endoscopy postoperatively may be warranted prior to use of the horses in experimental 410 studies as laryngeal hemiplegia may develop following the surgery. 


\section{Disclosure statement}

412

413 The authors declare no conflict of interest related to this report.

414 
416 1. Bone JF, Metcalfe J, Parer JT: Surgical preparation of a carotid loop in sheep. Am $417 \quad J$ Vet Res 1962;23:1113-1116

418 2. Tavernor WD: Technique for the subcutaneous relocation of the common carotid $419 \quad$ artery in the horse. Am J Vet Res 1969;30:1881-1884

420 3. Dueck R, Schroeder JP, Parker HR, et al: Carotid artery exteriorization for 421 percutaneous catheterization in sheep and dogs. Am J Vet Res 1982;43:898-901

422 4. Lagutchik MS, Sturgis JW, Martin DG, et al: Review of the carotid artery loop 423 procedure in sheep. J Invest Surg 1992;5:79-89

424 5. Orsini JA, Roby KA: Modified carotid artery transposition for repetitive arterial 425 blood gas sampling in large animals. J Invest Surg 1997;10:125-128

426 6. Prassinos N, Raptopoulos D, Adamama-Moraitou K, et al: Comparison of three 427 different techniques for subcutaneous relocation of the carotid artery in small 428 ruminants. J Vet Med A Physiol Pathol Clin Med 2001;48:15-21

429 7. Tate LP, Corbett WT, Foreman JH, et al: Instrumentation of exercising 430 thoroughbreds to determine blood gas tensions and acid-base status. Vet Surg $1993 ; 22: 171-176$

8. Benredouane K, Lepage O: Trans-arterial coil embolization of the internal carotid 433 artery in standing horses. Vet Surg 2012;41:404-409

434 9. Dixon P, Robinson E, Wade JF (eds): Workshop summary, in Proc Havemeyer 435 Workshop on Equine Recurrent Laryngeal Neuropathy, Stratford-upon-Avon, 436 UK, 2004. pp 93-97 
437 10. Butler HC: Subcutaneous relocation of the carotid artery for experimental $438 \quad$ purposes. Am J Vet Res 1962;23:165-166

439 11. Lorenz MD, Coates JR, Kent M: Disorders of the face, tongue, esophagus, larynx 440 and ear, in Lorenz MD, Coates JR, Kent M (eds):Handbook of Veterinary 441 Neurology (ed 5). St Louis, MO, Saunders, 2011. pp 282-306

442 12. Dixon PM, McGorum BC, Railton DI, et al: Laryngeal paralysis: A study of 375 443 cases in a mixed-breed population of horses. Equine Vet J 2001;33:452-458

444 13. Grant GA, Goodkin R, Kliot M: Evaluation and surgical management of 445 peripheral nerve problems. Neurosurgery 1999;44:825-39; discussion 839-840

446 14. MacKay RJ: Peripheral nerve injury, in Auer JA, Stick JA (eds): Equine Surgery 447 (ed 4). St Louis, MO, Saunders, 2012. pp 720-727

448 15. Harkins JD, Mitchell L, Hackett RP, et al: Catheterisation of carotid artery in 449 horses using ultrasonography. Equine Vet J 1992;24:480-481

450 16. Maninchedda U, Lepage OM, Gangl M, et al: Percutaneous ultrasound-guided 451 arterial angiography for transarterial coil placement in anesthetized and standing $452 \quad$ horses. Vet Surg 2015;44:322-327 
$454 \quad$ Figure legends

455

456 Figure 1: A photograph of the surgical area with drawn lines indicating the location of

457 the incision in relation to the jugular vein and the common carotid artery on the right side

458 of the neck. The blue line represents the jugular vein, the red line the common carotid 459 artery, and the yellow line the skin incision.

460

461 Figure 2: A schematic illustration of the incision and the approach to the carotid sheath.

462 The horse's head is to the right.

463

464 Figure 3: Surgical area on the right side of the neck, with the horse's head to the right.

465 The common carotid artery is elevated to the incision with Penrose drains and the muscle

466 layers are partially closed. The surgeon is holding the suture of the superficial part and

467 fascia of the brachiocephalicus muscle before tying the knot.

468

469

470 
471 Table

472 Table 1: Summary of the results in the individual horses.

$473 *$ Common carotid artery

$474 * *$ The outcome was considered successful if the subcutaneously translocated common

475 carotid artery was catheterized repeatedly without difficulties in the experiments

476 following the surgical procedure

477

\begin{tabular}{|c|c|c|c|c|c|}
\hline Horse & $\begin{array}{l}\text { Ligation of } \\
\text { the } \\
\text { branches of } \\
\text { the CCA }\end{array}$ & $\begin{array}{l}\text { Use of a } \\
\text { stent } \\
\text { bandage }\end{array}$ & $\begin{array}{l}\text { Closure of the } \\
\text { subcutaneous } \\
\text { layer }\end{array}$ & Complications & Outcome \\
\hline $\begin{array}{l}\text { Horse } \\
1\end{array}$ & No & No & No & $\begin{array}{l}\text { Right-sided } \\
\text { laryngeal } \\
\text { hemiplegia }\end{array}$ & Successful $^{* *}$ \\
\hline $\begin{array}{l}\text { Horse } \\
2\end{array}$ & No & No & No & None & Successful $^{* *}$ \\
\hline $\begin{array}{l}\text { Horse } \\
3\end{array}$ & No & No & No & $\begin{array}{l}\text { Surgical site } \\
\text { infection }\end{array}$ & $\begin{array}{l}\text { Loss of } \\
\text { patency of } \\
\text { the } \\
\text { translocated } \\
\text { CCA }^{*}\end{array}$ \\
\hline $\begin{array}{l}\text { Horse } \\
4\end{array}$ & No & Yes & Yes & $\begin{array}{l}\text { Excessive } \\
\text { periarterial } \\
\text { fibrosis }\end{array}$ & $\begin{array}{l}\text { Loss of } \\
\text { patency of } \\
\text { the } \\
\text { translocated } \\
\text { CCA }^{*}\end{array}$ \\
\hline $\begin{array}{l}\text { Horse } \\
5\end{array}$ & No & Yes & No & $\begin{array}{l}\text { Right-sided } \\
\text { laryngeal } \\
\text { hemiplegia }\end{array}$ & Successful $^{* *}$ \\
\hline $\begin{array}{l}\text { Horse } \\
6\end{array}$ & Yes & Yes & No & None & Successful $^{* *}$ \\
\hline $\begin{array}{l}\text { Horse } \\
7\end{array}$ & No & Yes & No & None & Successful $^{* *}$ \\
\hline $\begin{array}{l}\text { Horse } \\
8\end{array}$ & Yes & Yes & No & None & Successful $^{* *}$ \\
\hline
\end{tabular}


478 